\title{
Individuals with opioid dependence who leave drug treatment have higher overdose and death rates than those still in treatment
}

Digiusto E, Shakeshaft A, Ritter A, et al. Serious adverse events in the Australian National Evaluation of Pharmacotherapies for Opioid Dependence (NEPOD). Addiction 2004;99:450-60.

\section{Among people with opioid dependence, what is the estimated rate of serious adverse events during and after pharmacotherapy?}

\section{METHODS}

Design: Prospective longitudinal study (combined data from 12 trials included in the Australian National Evaluation of

Pharmacotherapies for Opioid Dependence).

Follow up period: Maximum of six months after ending treatment (the last date medication was taken)

Setting: Hospital, community, and GP settings in Australia; time frame not stated.

으 People: From a total of 1244 participants, 280 (23\%) were stable in methadone treatment when entering the trials, and 964 $(77 \%)$ were regular heroin users (assessed as opioid dependent by DMS-VI criterial.

$\Delta$

Risk factors: Participants entered a drug maintenance treatment. Individuals were given either an opioid agonist (methadone, $n=403$; buprenorphine, $n=402$, or levo-alpha acetyl methado (LAAM), $n=115$ ) or an opioid antagonist (naltrexone, $n=324$ ).

Outcomes: Serious adverse life events, as defined by the Australian Therapeutic Goods Administration. Recorded events included: heroin overdose, general illness accidents/injuries, other drug reactions, events of a psychiatric nature, pregnancy related events, or admission for inpatient detoxification. Data were collected during routine contact with clinicians and recorded in case notes. Incidence data were standardised as rates per 100 person years of observation.

\section{MAIN RESULTS}

Ninety six serious adverse events (SAEs) were recorded, including 32 heroin overdoses (five agonist-all buprenorphine, 27 naltrexone), 15 hospital admissions for detoxification (16 naltrexone, one agonist), eight adverse drug reactions (four naltrexone, four agonist), eight psychiatric events (one naltrexone, seven agonist); the

Table Serious adverse event rates per 100 person years $(95 \% \mathrm{Cl})$

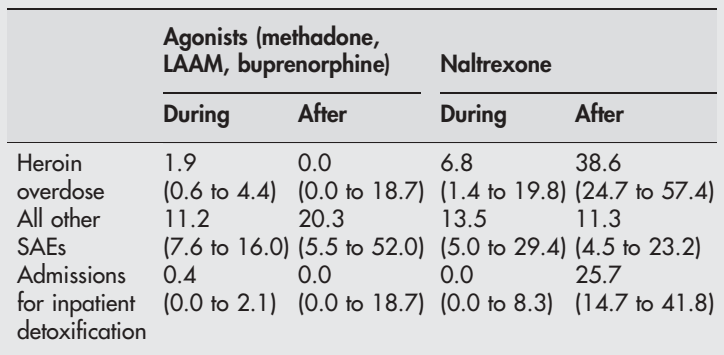
UNSW, Sydney, Australia; Erol@accsoft.com.au

Sources of funding: the Commonwealth Department of Health and Aged care. remaining 31 events were generally illnesses, accidents or injuries, or pregnancy related events. People taking naltrexone had eight times the rate of overdose after leaving treatment compared with agonist treated participants; $44 \%$ of these occurring within two weeks of leaving treatment. Rates of all other SAEs were similar between groups, and between those in treatment and those who hade left (see table). Five deaths occurred, all in people leaving treatment (rate of six per 100 person years). Total SAE rates between agonist (20 per 100 person years) and naltrexone group (14 per 100 person years) were not significantly different. Total SAE and death rates were higher post-treatment than during treatment (three and 19 times, respectively).

\section{CONCLUSIONS}

Individuals who have left drug treatment for opioid dependence have higher overdose and death rates than those still in treatment. This could be partly a participant self-selection effect rather than entirely a protective effect of pharmacotherapy. Clinicians should alert people taking naltrexone regarding risks of heroin overdose.

\section{Commentary}

$\mathrm{D}$ igiusto ef al's study focuses on serious adverse events (SAEs) occurring among participants of trials examining opioid agonist/ partial agonist versus antagonist (naltrexone) pharmacotherapies. SAEs have been reported among people treated with naltrexone. ${ }^{1}$ There is no evidence of significant differences in SAE rates between methadone, levo-alpha acetyl methadol (LAAM) and buprenorphine treatment, while it is known that opioid overdoses often occur after people leave treatment. $^{2}$

The present study provides the most comprehensive analysis to date of SAEs associated with pharmacotherapy for opioid dependence because: (1) a large number of participants were included; (2) all common opioid pharmacotherapies were evaluated, and (3) SAE rates during treatment were compared with rates observed after the participants left treatment.

Opioid overdoses were the most frequent SAE recorded. This occurred in participants treated with naltrexone and buprenorphine, and mainly after they had stopped taking naltrexone. Clinical consequences of this study can be summarised as follows: people treated with naltrexone or buprenorphine must be advised about the consequences of heroin use; an unusually high dose of heroin may induce overdose as opioid tolerance decreases during abstinence from heroin, either as a result of treatment (naltrexone, buprenorphine) or withdrawal from treatment. Also, risk of overdose may increase if sedative drugs are co-administered (benzodiazepines, antipsychotics, hypnotics, and anticonvulsant agents are commonly used in people with substance abuse). It is imperative that clinicians should advise people being treated with naltrexone and buprenorphine about the consequences of heroin use, especially when they leave detoxification units or are released from prison because during that period their opioid tolerance is very low and craving for heroin could be high. Finally, clinicians should consider the use of methadone instead of buprenorphine in people with high risk of suicide (people with antisocial, borderline personality disorders or affective disorders).

Gabriel Rubio, MD

Alcoholism Program, "Retiro" Mental Health Service, Madrid, Spain

1 Miotto K, McCann MJ, Rawson RA, et al. Overdose, suicide attempts and death among a cohort of naltrexone-treated opioid addicts. Drug Alcohol Depend 1997;45:131-4.

2 Clark N, Lintzeris N, Gijsbers A, et al. LAAM maintenance vs methadone maintenance for heroin dependence. In: The Cochrane Library, Issue 3. Oxford: Update Software. 